\title{
Spatial distribution of dust in the shell elliptical NGC 5982
}

\author{
C. del Burgo ${ }^{1}$, D. Carter $^{2}$, and G. Sikkema ${ }^{3}$ \\ 1 School of Cosmic Physics, Dublin Institute for Advanced Studies, Dublin 2, Ireland \\ e-mail: cburgo@cp.dias.ie \\ 2 Astrophysics Research Institute, Liverpool John Moores University, 12 Quays House, Egerton Wharf, Birkenhead, CH41 1LD, UK \\ 3 Kapteyn Astronomical Institute, University of Groningen, PO Box 800, 9700 AV Groningen, The Netherlands
}

Received 22 May 2007 / Accepted 8 October 2007

ABSTRACT

\begin{abstract}
Aims. Shells in Ellipticals are peculiar faint sharp edged features that are thought to be formed by galaxy mergers. We determine the shell and dust distributions, and colours of a well-resolved shell and the underlying galaxy in NGC 5982, and compare the spatial distributions of the dust and gas phases, in order to investigate the origin of shells and dust in NGC 5982.

Methods. We use Spitzer data in the wavelength range from 3.6 to $160 \mu \mathrm{m}$ and HST/ACS optical data. Surface photometry, galaxy models and residual images are determined using IRAF task ELLIPSE and GALPHOT task ELLIPFIT. After subtracting the galaxy models, residual images are used to identify the shells. Excess emission is identified by subtracting a scaled stellar photosphere emission template. Colours [3.6]-[4.5], [3.6]-[8.0] and [5.8]-[8.0] of the underlying galaxy and [5.8]-[8.0] of the excess emission are obtained using techniques similar to those of Pahre et al. The [3.6]-[4.5] and $V-I$ colours of a well-resolved shell are determined using techniques of Sikkema et al.

Results. Shells are visible in the $3.6 \mu \mathrm{m}$ image, fainter at $4.5 \mu \mathrm{m}$ and undetected at $5.8 \mu \mathrm{m}$. We find two new shells, the outermost identified so far in NGC 5982. All shells apart from shell 24 are on the photometric major axis. Shell 24 is the only one for which reliable colours are obtained. It has colours [3.6]-[4.5] and $V-I$ bluer than the underlying galaxy. Excess emission at 4.5, 5.8 and $8 \mu \mathrm{m}$ is widely extended. The ratio of excess to total emission decreases towards the centre, which could be related to a lower mass loss rate from AGB stars. The [3.6]-[4.5] colour indicates a major contribution from late-type (K2-M0III) stars towards the centre. Dust traced by $24 \mu \mathrm{m}$ emission follows the stellar spatial distribution, supporting a circumnuclear origin. We find extended $160 \mu \mathrm{m}$ emission from cold dust, possibly forming a disk inclined to the principal axes, with a mass of a few $10^{5} M_{\odot}$. The warmer big grains and/or smaller grains traced by the $70 \mu \mathrm{m}$ emission are confined to a small region elongated along the minor axis and shifted a few $\mathrm{kpc}$ from the centre.

Conclusions. We detect for the first time shells from mid-infrared data. The very different distributions of dust, warm gas and HI gas together with the presence of shells and a kinematically decoupled core suggest a minor merger in NGC 5982.
\end{abstract}

Key words. galaxies: elliptical and lenticular, cD - galaxies: photometry - galaxies: ISM - galaxies: formation - galaxies: interactions

\section{Introduction}

Shell galaxies (Malin \& Carter 1980, 1983) are elliptical galaxies with faint, often sharp edged features in their envelopes, which are generally interpreted as the remnants of mergers of low mass, low velocity dispersion galaxies with the elliptical (Quinn 1984; Hernquist \& Quinn 1988). The study of the structure and morphology of these features is useful in interpreting the parameters of minor mergers, and the importance of mergers in determining the present day structure of ellipticals (Dupraz \& Combes 1986; Nulsen 1989).

The interstellar medium (ISM) in ellipticals mostly consists of X-ray emitting hot gas $\left(T \sim 10^{7} \mathrm{~K}\right)$ but it may also contain significant amounts of warm and cold gas and dust. Recent studies show that approximately $70 \%$ of ellipticals harbour HI gas (Morganti et al. 2006); a similar proportion contains warm gas (Sarzi et al. 2006). For a similar galaxy sample, Combes et al. (2007) find a CO detection rate of $28 \%$ in the central regions. The far-infrared emission in ellipticals gauges the bulk of dust, which has masses from $10^{5} M_{\odot}$ to $10^{7} M_{\odot}$ (Temi et al. 2004, 2007).
Our knowledge of the far-infrared emission from earlytype galaxies is largely based on Infrared Astronomical Satellite (IRAS) data at 12, 25, 60 and $100 \mu \mathrm{m}$. IRAS was successful in detecting emission from dust with a wide range of temperatures in many bright elliptical galaxies (Knapp et al. 1989). These observations were limited, however, by sensitivity, angular resolution ( $\sim 4^{\prime}$ on the sky) and the lack of filterbands to gauge and resolve the emission from cold dust. Infrared Space Observatory ISO improved this situation, providing $\sim 1.5^{\prime}$ angular resolution observations at 150 and $200 \mu \mathrm{m}$ which are more sensitive to the peak emission from ellipticals. It also included filterbands comparable to IRAS and mid-infrared imaging at an angular resolution of a few arcseconds, to study the spatial distribution of the mid-infrared carriers (see Ferrari et al. 2002; Xilouris et al. 2004). ISO could not always resolve the structure in elliptical galaxies, which may have emission from Active Galactic Nuclei (AGNs), dense clouds, circumstellar dust and diffuse interstellar dust (Temi et al. 2004). The modern Spitzer instruments IRAC (filterbands at 3.6, 4.5, 5.8, 8,0 $\mu \mathrm{m}$ ) and MIPS (broad filterbands at $24,70,160 \mu \mathrm{m})$ offer unprecedent sensitivity and angular resolution to much better differentiate the different dust components in a sample of ellipticals with a wide luminosity range 
(see, e.g., Pahre et al. 2004, hereinafter PAFW04). Spitzer IRS is used to observe the spectral energy distribution (SED) of the mid-infrared carriers in early-type galaxies (Kaneda et al. 2005; Bressan et al. 2006).

It is thought that dust is produced by evolving red giant stars in the old stellar population typical of ellipticals, and it is dispersed into the interstellar medium (ISM). Conversely, dust is destroyed by sputtering in the host interstellar hot gas of ellipticals. During their sputtering lifetimes big dust grains in the ISM are heated by the interstellar radiation field and thermal electron impacts to temperatures that radiate in the far-infrared (see Temi et al. 2004, and references therein). Small optically thick clouds have been also observed in $\approx 50 \%$ of bright ellipticals (van Dokkum \& Franx 1995; Lauer et al. 2005). These clouds are likely formed from dusty gas expelled from stars in the central Kiloparsec of ellipticals (Mathews \& Brighenti 2003). Dust can be also produced in galaxy mergers and it is largely accepted that ellipticals are formed by mergers. However, it is not straightforward to explain all kinematical and structural properties in ellipticals by the current merger models.

NGC 5982 is a shell galaxy with regular major axis shells (Sikkema et al. 2007, hereinafter SCP07), which identifies it as type I in the classification scheme of Prieur (1988). The redshift is $c z=3017 \mathrm{~km} \mathrm{~s}^{-1}$ (Smith et al. 2000), which corresponds to a distance $D=43 \mathrm{Mpc}$ assuming $H_{0}=70 \mathrm{~km} \mathrm{~s}^{-1} \mathrm{Mpc}^{-1}$. NGC 5982 is the brightest galaxy of LGG 402 with 4 members (García 1993). There is a possible very low activity level LINER type nucleus (Ho et al. 1997). This galaxy harbours a Kinematically Decoupled Core (KDC: Wagner et al. 1988), and probably a rotating central disk (Emsellem et al. 2004). Outside the core, the galaxy is slowly rotating (Emsellem et al. 2004) and presents central boxy isophotes (SCP07).

This paper presents a photometric analysis of the shells and the underlying galaxy in the elliptical (E3) galaxy NGC 5982 from Spitzer infrared data, and compares this with our earlier analysis of HST/ACS $V$ - and $I$-band photometric data (SCP07). The observations and data processing are presented in Sect. 2. Section 3 is devoted to the analysis of the data. The results and their interpretation are presented in Sect. 4. Section 5 contains the discussion of our results, and finally, Sect. 6 presents our conclusions.

\section{Observations and data processing}

We use public Spitzer and Hubble Space Telescope (HST) data. The first were retrieved from the Spitzer Data Archive using the Archive Tool Leopard. The HST observations belong to our programme to study shell ellipticals (GO9399, PI: D. Carter) and are described in two earlier publications (Sikkema et al. 2006; SCP07).

\subsection{IRAC observations}

NGC 5982 has been observed using the Infrared Array Camera (IRAC; Fazio et al. 2004) on the Spitzer Space Telescope (Werner et al. 2004) during the campaign IRAC005700 of GO programme number 3403 (PI: J. Surace). NGC 5982 was imaged using 14 exposures of $26.8 \mathrm{~s}$ (effective exposure time per pixel) centred on different positions with the four IRAC filterbands at 3.6, 4.5, 5.8 and $8 \mu \mathrm{m}$. We use the standard pipeline (version 14.0) post Basic Calibrated Data (post-BCD) products. The mosaics resulting from the addition of the 14 exposures have effective areas between 103 and $104 \operatorname{arcmin}^{2}$ and they are less noisy in an oversampled $7^{\prime} \times 7^{\prime}$ square region centred on NGC 5982. The image mosaics at 3.6 and $5.8 \mu \mathrm{m}$ roughly sample that square region and a similar-sized region to the ENE side; those at 4.5 and $8.0 \mu \mathrm{m}$ cover the square region and a similar sized region to the WSW side. These image mosaics are large enough to derive reliable values of the sky background to subtract from the images. The angular resolution of the IRAC images is $F W H M \sim 1.8^{\prime \prime}$.

The existing calibration of IRAC data is based on pointsource observations (Fazio et al. 2004). Extended sources require a different calibration. We use a similar approach to PAFW04 in order to determine instrumental magnitudes relative to Vega. The aperture corrections of PAFW04 are based on large aperture photometry of NGC 777 assuming that it has no dust emission and that the 2MASS near-infrared and IRAC colours match those corresponding to a MOIII star. This is a suitable stellar type to match the SED from an early-type galaxy in the $K$-band (Frogel et al. 1978). We also use the aperture corrections of Reach et al. (2005, hereinafter RMC05) and compare the results for both aperture corrections. The most significant differences are for the aperture correction at $5.8 \mu \mathrm{m}$. See Sect. 3.2 for a detailed discussion.

\subsection{MIPS observations}

NGC 5982 was also observed using the Multiband Imager Photometer (MIPS) (Rieke et al. 2004) on the Spitzer Space Telescope in three filterbands centred at 24,70 and $160 \mu \mathrm{m}$ during the campaign MIPS004600 of GO programme number 3403 (PI: J. Surace). Again we use the standard pipeline (version 14.0) post Basic Calibrated Data (post-BCD) products. At $24 \mu \mathrm{m}$, with $2.45^{\prime \prime} \mathrm{pix}^{-1}$, the resulting mosaic centred on the galaxy has a field of view of $7.4^{\prime} \times 8.2^{\prime}$. In addition there are two images with fields of view of $5.6^{\prime} \times 8.0^{\prime}$ and $6.1^{\prime} \times 10.1^{\prime}$, to the NE and SW of the galaxy, respectively, that we used to determine the sky background. At $70 \mu \mathrm{m}$, the pixel size is $4^{\prime \prime}$ and the useful area is $1.2^{\prime} \times 2.9^{\prime}$. At $160 \mu \mathrm{m}$ the pixel size is $8^{\prime \prime}$ and the observed area centred on the galaxy is $6.3^{\prime} \times 5.5^{\prime}$. The sky background is estimated at each wavelength and subtracted from the corresponding image.

The angular resolution of the MIPS images is FWHM $\sim 6^{\prime \prime}$, $\sim 18^{\prime \prime}$ and $\sim 40^{\prime \prime}$ at 24,70 and $160 \mu \mathrm{m}$, respectively.

The same observations have already been used by Temi et al. (2007) to derive luminosities of NGC 5982 at 24, 70 and $160 \mu \mathrm{m}$ of $L_{24}=10^{40.86} \mathrm{erg} \mathrm{s}^{-1}, L_{70}=10^{40.92} \mathrm{erg} \mathrm{s}^{-1}$ and $L_{160}=10^{40.66} \mathrm{erg} \mathrm{s}^{-1}$.

\subsection{HST/ACS data}

HST ACS_WFC camera data (Pavlovsky et al. 2004) obtained with the filters $F 606 W$ ( $V$ band) and $F 814 W$ ( $I$ band) are used here. For a detailed description of the observations and the data processing see Sikkema et al. (2006) and SCP07, here we just provide a summary. The field of view was of $202^{\prime \prime} \times 202^{\prime \prime}$, with a pixel size of 0.049 arcsec. The exposure times were of 1314 and $1020 \mathrm{~s}$ in the $V$ - and $I$-bands, respectively. The $80 \%$ completeness levels were of 26.08 and $24.80 \mathrm{mag}$ in $V$ - and $I$-bands, respectively. In this paper we use the $V$ - and $I$-band images to extract shell profiles in these filterbands (see Sect. 4.4) that were not presented in SCP07. We use the technique introduced by SCP07 to determine the surface brightness locally in wedges for the underlying galaxy and the shells. 

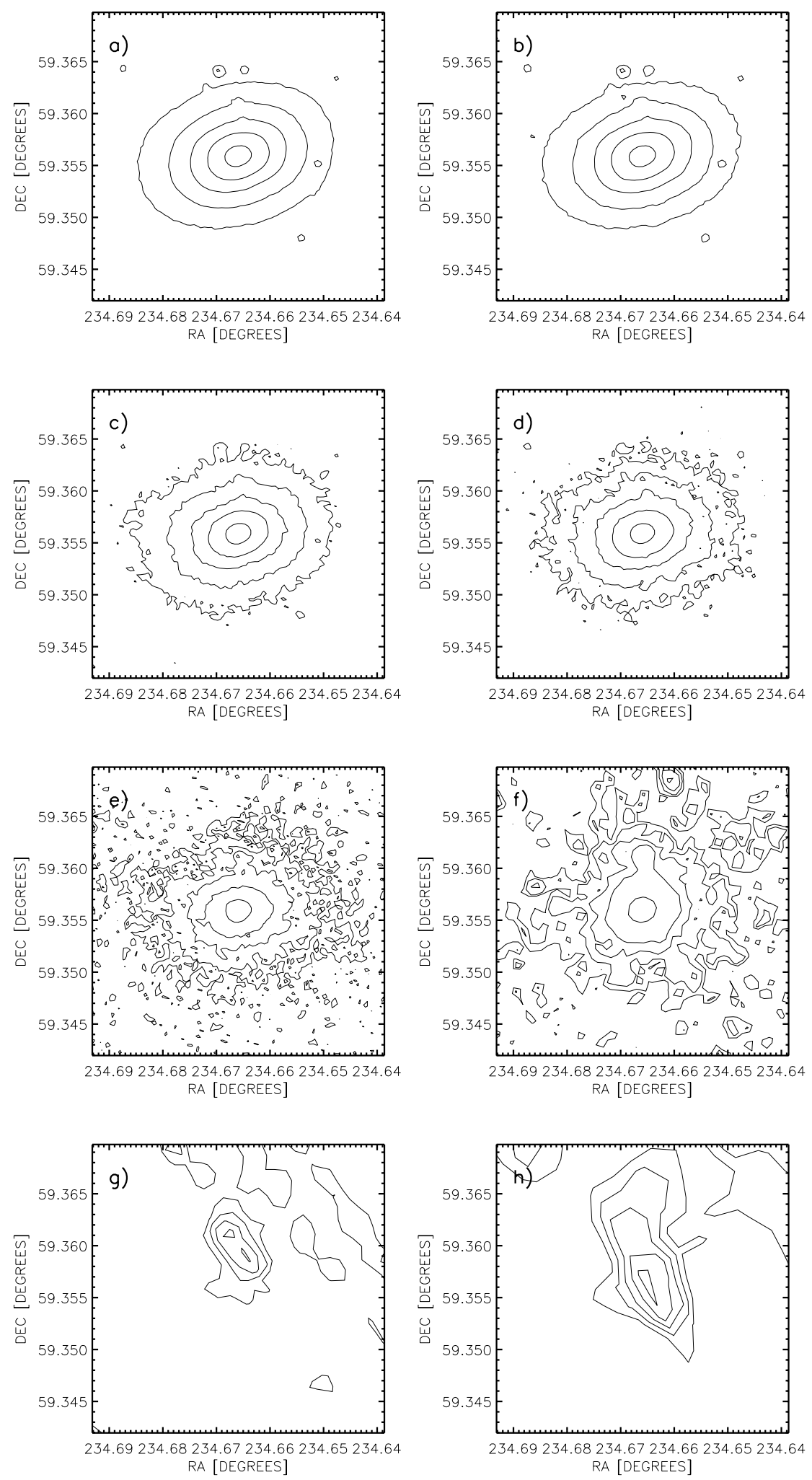

Fig. 1. Surface brightness at $3.6 \mu \mathrm{m}$ a), $4.5 \mu \mathrm{m} \mathrm{b}), 5.8 \mu \mathrm{m}$ c) and $8.0 \mu \mathrm{m} \mathrm{d}$ ); surface brightness at $8.0 \mu \mathrm{m}$ e); contour emission maps at $24 \mu \mathrm{m} \mathrm{f)}$, $70 \mu \mathrm{m} \mathrm{g}$ ) and $160 \mu \mathrm{m} \mathrm{h}$ ) of NGC 5982. FWHM at 3.6, 4.5, 5.8 and $8 \mu \mathrm{m}$ is $\sim 1.8^{\prime \prime} ; F W H M \sim 6^{\prime \prime}, \sim 18^{\prime \prime}$ and $40^{\prime \prime}$ at $24 \mu \mathrm{m}, 70 \mu \mathrm{m}$ and $160 \mu \mathrm{m}$, respectively.

\section{Analysis}

\subsection{Mid- and far-infrared emission images}

Surface brightness images of NGC 5982 of $100^{\prime \prime} \times 100^{\prime \prime}$ in size on the sky are presented in Figs. $1 \mathrm{a}-\mathrm{d}, \mathrm{f}-\mathrm{h}$ corresponding to the emission at $3.6,4.5,5.8,8.0,24,70$ and $160 \mu \mathrm{m}$, respectively. At first view, the surface brightness distribution at 3.6, 4.5, 5.8 and $8.0 \mu \mathrm{m}$ is very similar. The $24 \mu \mathrm{m}$ surface brightness distribution is also very similar to them, but very different from those at 70 and $160 \mu \mathrm{m}$. The latter two are elongated along the 

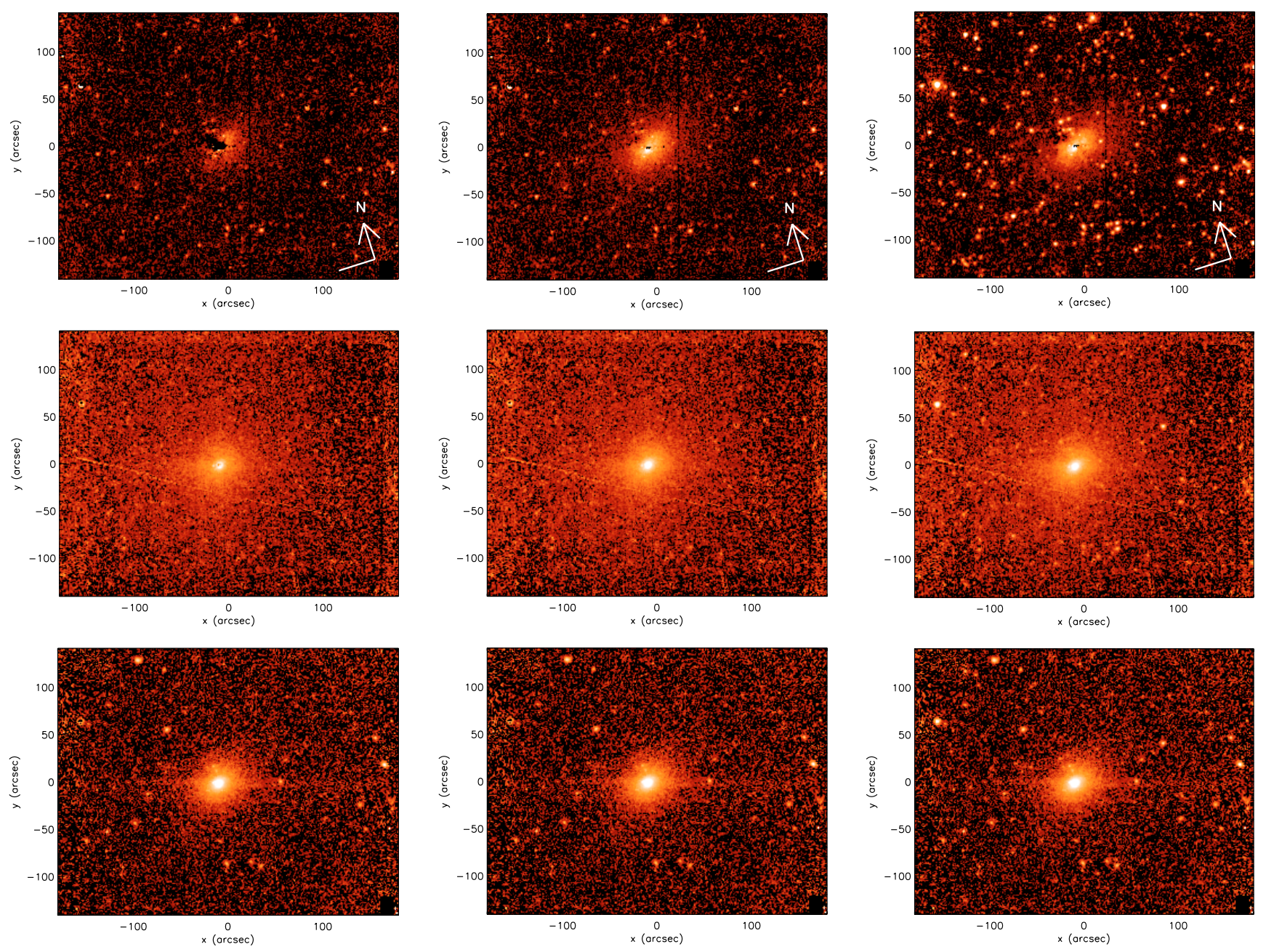

Fig. 2. From top to bottom excess emission at 4.5, 5.8 and $8 \mu \mathrm{m}$. First and second column results are obtained using the observed image at $3.6 \mu \mathrm{m}$ as stellar emission template, and the PAFW04 and RMC05 aperture corrections, respectively. Third column contains the results corresponding to the galaxy model at $3.6 \mu \mathrm{m}$ as stellar emission template and the RMC05 aperture corrections. The surface brightness scale is the same for each wavelength; it ranges from 0.0005 to 0.5 times the maximum (in logarithmic scale) corresponding to the second column. North is indicated by the arrow; East is 90 degrees anticlockwise in the top panels.

photometric minor axis of the galaxy, with the $70 \mu \mathrm{m}$ emission not resolved along the perpendicular axis. There is a double peak emission at $70 \mu \mathrm{m}$ that is shifted with respect to the photometric centre, with the bulk of the emission to the North. The $160 \mu \mathrm{m}$ emission is more extended than that at $70 \mu \mathrm{m}$, with emission to both North and South of the centre.

The sky background is subtracted from each image. Hereafter, we refer to each image corrected for the sky background as galaxy emission, observed emission, or simply observed image.

\subsection{Excess emission at 4.5, 5.8 and $8.0 \mu \mathrm{m}$}

A scaled stellar photosphere emission template is subtracted from the 4.5, 5.8 and $8.0 \mu \mathrm{m}$ images to obtain any excess emis$\operatorname{sion}^{1}$ at these wavelengths. We follow a similar approach to PAFW04 but using the image at $3.6 \mu \mathrm{m}$ only instead of a combination of the images at 3.6 and $4.5 \mu \mathrm{m}$ as our stellar template. We also use the GALPHOT galaxy model at $3.6 \mu \mathrm{m}$ (see next section) as a stellar template. For each template, we use the aperture

1 Other authors refer to these images as "dust images" or "nonstellar" images (see PAFW04). corrections provided by PAFW04 as well as those of RMC05 and compare the results. The stellar template is scaled to match the corresponding theoretical colour of M0III stars, before being subtracted from the observed emission at 4.5, 5.8 and $8.0 \mu \mathrm{m}$ (PAFW04).

Figure 2 shows the excess emission at 4.5, 5.8 and $8.0 \mu \mathrm{m}$ in NGC 5982 for three of the four possible combinations of templates and aperture corrections. Figure 1e shows the contours of the excess emission of NGC 5982 at $8.0 \mu \mathrm{m}$ obtained with the image at $3.6 \mu \mathrm{m}$ as stellar template and the RMC05 aperture corrections for comparison with the observed images shown in Fig. 1.

We highlight some remarks resulting from our comparison of the excess emission obtained with the two stellar templates combined with each of the aperture corrections:

- The excess emission increases toward longer wavelengths. At each wavelength, the ratio between the excess emission and the total emission varies with the radius. These ratios are higher when using the aperture corrections of RMC05, especially at $5.8 \mu \mathrm{m}$.

- Excluding a few central pixels, there are no significant differences between the excess emission images obtained using the two stellar templates for a given aperture correction. 
Note also that point-like sources entirely remain in the excess emission maps when subtracting the scaled GALPHOT galaxy model.

- There is a central dark spike that is prominent at $4.5 \mu \mathrm{m}$ (see Fig. 2, top-left) and less visible at $5.8 \mu \mathrm{m}$ (see Fig. 2, middleleft) when using the aperture corrections of PAFW04. This dark spike contrasts less with the surroundings when using the aperture corrections of RMC05.

- We have verified that this spike is not related to a crosscorrelation effect due to any difference in the IRAC instrumental profiles.

We also note that the present excess emission at 5.8 and $8.0 \mu \mathrm{m}$ has a similar spatial distribution to that obtained using a combination of the images at 3.6 and $4.5 \mu \mathrm{m}$ as stellar emission template.

\subsection{Surface photometry: global parameters}

Surface photometry at wavelengths $3.6,4.5,5.8$ and $8.0 \mu \mathrm{m}$ is obtained using an ellipse fit with the task ELLIPFIT of GALPHOT (Jørgensen et al. 1992) and the task ELLIPSE from the IRAF STSDAS package. The latter is used to measure the flux using the same isophotal shapes defined from a reference image (i.e., the $3.6 \mu \mathrm{m}$ image). ELLIPFIT provides us a galaxy model and a table with the fitted parameters, including ellipticity $(\epsilon)$, position angle (PA), surface brightness $(\mu)$ and the coefficients $C 3, C 4, S 3$ and $S 4$ (Carter 1978) for different distances from the centre of the galaxy. Point-like sources, background galaxies, dust features, bright shells and artifacts are identified and masked by hand in an iterative way. Remaining faint shell structures have a negligible effect on the results. The best fits are obtained by allowing centre, PA and ellipticity to vary with radius. There is an excellent agreement between the values (e.g. of $\epsilon$ and PA) obtained using ELLIPSE and ELLIPFIT.

The inner region ( $r \lesssim 15-20^{\prime \prime}$ ) is poorly fitted compared with the rest of the galaxy, especially for the $3.6 \mu \mathrm{m}$ image. The differences between the observed image and galaxy model are up to $\sim 3 \%$. This fitting problem was also noted at optical wavelengths by SCP07, who suggested that it could be due to the KDC, which is $\sim 10^{\prime \prime}$ in size (Mc Dermid et al. 2006). Alternatively it may be related to the presence of dust or the high density of point-like objects in the innermost region of NGC 5982. The ellipticity gets significantly lower in the inner 4" of the galaxy and it was not possible to fit smoothly the central region. We obtain the best result using 6 harmonics. The values of the $\mathrm{C} 4$ coefficient for $a \gtrsim 5^{\prime \prime}$ are very similar to those determined from the HST/ACS I-band image (SCP07), and indicate a boxy structure (Carter 1978).

Global surface brightness profiles for NGC 5982 are shown by plotting $\mu$ for each fitted ellipse as function of semi-major axis $a$. Figures $3 \mathrm{a}, \mathrm{c}$ and d respectively shows $\mu, \epsilon$ and PA versus $a$ at $3.6 \mu \mathrm{m}$. To investigate the effect of the IRAC Point Source Response Function (PSF) $\left(F W H M \sim 1.8^{\prime \prime}\right)$ upon the surface brightness, ellipticity and position angle profiles, we measure $\mu, \epsilon, \mathrm{PA}, S 4$ and $C 4$ in the HST/ACS $V$-band image and also in the image resulting from convolving it with the IRAC PSF at $3.6 \mu \mathrm{m}$. We show only the results for $\epsilon$ and PA (see Figs. 3c and $\mathrm{d}$, respectively). The ellipticity drops toward smaller radii partly as result of degradation of the angular resolution. The effect on PA is much smaller.
We use a Sérsic (1968) profile to model the luminosity at $3.6 \mu \mathrm{m}$. This profile is defined by the equation:

$\mu(r)=\mu_{\mathrm{e}}+c_{n}\left[\left(\frac{r}{r_{\mathrm{e}}}\right)^{1 / \mathrm{n}}-1\right]$

where $c_{n}=2.5(0.868 n-0.142)$ for $0.5<n<16.5$ (Caon et al. 1993).

Table 1 lists the least-squares fitted values for $\mu_{\mathrm{e}}, n$ and $r_{\mathrm{e}}$ for $1.2^{\prime \prime} \leq a \leq 100^{\prime \prime}$. The difference between the luminosity profile at $3.6 \mu \mathrm{m}$ and the fitted model is plotted in Fig. $3 \mathrm{~b}$.

\subsection{Residual images at 3.6, 4.5 and $5.8 \mu \mathrm{m}$ in NGC 5982}

We compute residual images from the difference between the observed image at 3.6, 4.5 and $5.8 \mu \mathrm{m}$ and the corresponding galaxy models at these wavelengths obtained from ELLIPFIT (see previous section). Figure 4 (left) shows the residual image at $3.6 \mu \mathrm{m}$ where faint shell-like structures are observed. The innermost shells are much less resolved in the Spitzer images than in the $R$-band (see Fig. 4, right) and the HST/ACS images. All the shells are much fainter at longer wavelengths. The residual image at $5.8 \mu \mathrm{m}$ does not show any shells. We note that the innermost shells are in a region where some $4.5 \mu \mathrm{m}$ excess emission is observed (see Figs. 2 (top) and 4).

Residual images are only used to identify and locate the shells. For the determination of the shell profiles and colours we work within wedges as in SCP07 to avoid the effect of large scale fluctuations in the sky background that affect the extraction of faint shell properties.

\subsection{Colour profiles}

Colour profiles [3.6]-[4.5], [3.6]-[8.0], [5.8]-[8.0] for the underlying stellar emission and the [5.8]-[8.0] colour for the excess emission (see Sect. 3.2) are determined using the isophotal shapes obtained from the $3.6 \mu \mathrm{m}$ image. The colours are, therefore, measured for the same regions of the galaxy. Figures $3 \mathrm{e}-\mathrm{g}$ show the colours [3.6]-[4.5], [3.6]-[8.0] and [5.8]-[8.0], respectively, using the RMC05 aperture corrections. Figure $3 \mathrm{~g}$ also shows the [5.8]-[8.0] colour of the excess emission obtained from the PAFW04 aperture corrections. The colour profiles are very sensitive to the sky background subtracted. Colours [3.6]-[8.0] and [5.8]-[8.0] are especially sensitive to the sky background emission at $8.0 \mu \mathrm{m}$ that may modify the colour profile from $a \sim 40^{\prime \prime}$. Colours are also sensitive to the aperture corrections (see Sect. 4.1), most significantly in the case of the [5.8]-[8.0] colour of the excess emission (see Fig. 3g). However, the differences in this colour using the two different stellar templates (see Sect. 3.2) are not significant. Azimuthally averaged surface brightness profiles practically wash away the shells visible in Fig. 4. The shells are too faint in NGC 5982 to affect the colour profiles (see Figs. $3 \mathrm{e}-\mathrm{g}$ ). As mentioned above, we use the technique of SCP07 based on wedges to extract faint shell colours (see Sect. 4.4).

\section{Results and data interpretation}

\subsection{Excess emission}

The isophotes of the excess emission at 5.8 and $8.0 \mu \mathrm{m}$ show a slightly different orientation of the major axis in comparison with the total emission (i.e., stellar plus excess emission) at these wavelengths (see Figs. 1d and e). The emission profiles at 5.8 


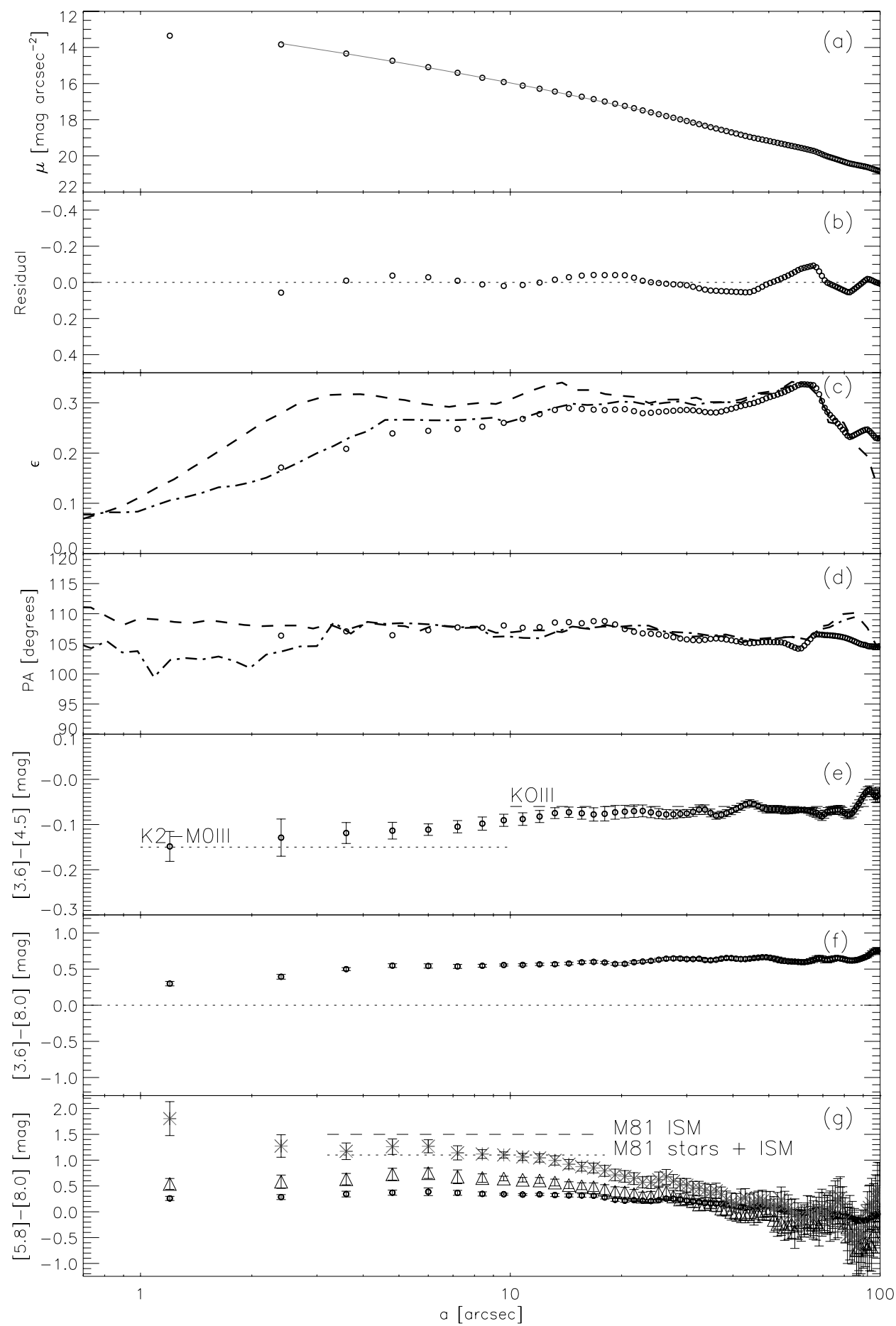

Fig. 3. Global parameters for NGC 5982: a) surface brightness at $3.6 \mu \mathrm{m}$ corrected for background (the continuous line is a Sérsic profile fit); b) difference between the $3.6 \mu \mathrm{m}$ surface brightness profile and the Sérsic fit; c) ellipticity corresponding to the $3.6 \mu \mathrm{m}$ image (points), the ACS/HST $V$-band image (as derived in SCP07; dashed line), the ACS/HST $V$-band image convolved with the IRAC PRF at $3.6 \mu \mathrm{m}$ (dash-dotted line); d) position angle PA (symbols as in previous panel); e) [3.6]-[4.5] colour for the underlying galaxy as well as the values for the KOIII (long-dashed line) and K2-M0III (short dashed line) stars as indicated by PAFW04; f) [3.6]-[8.0] colour for the underlying galaxy; g) [5.8]-[8.0] colour for the underlying galaxy (small circles) and for the excess emission using the aperture corrections of RMC05 (triangles) and PAFW04 (asterisks). Values corresponding to M 81 are also indicated (Willner et al. 2004).

and $8 \mu \mathrm{m}$ are significantly different from those at $3.6 \mu \mathrm{m}$. This is due to the observed excess emission at 5.8 and $8 \mu \mathrm{m}$. For each wavelength, the surface brightness ratio between the excess emission and the total emission varies with the radius. The change is different for the two aperture corrections used here. If applying those of PAFW04, the excess emission at $8 \mu \mathrm{m}$ is $\sim 17 \%$ and $\sim 37 \%$ of the total emission in the centre and for radii between $\sim 4^{\prime \prime}$ and $\sim 25^{\prime \prime}$ on the sky, respectively. These values are 3\% higher when using the RMC05 aperture corrections. At $5.8 \mu \mathrm{m}$, the values in the centre and outer region are respectively of $\sim 5 \%$ and $\sim 20 \%$ when using the PAFW04 aperture corrections. The values are 10\% higher when using the RMC05 

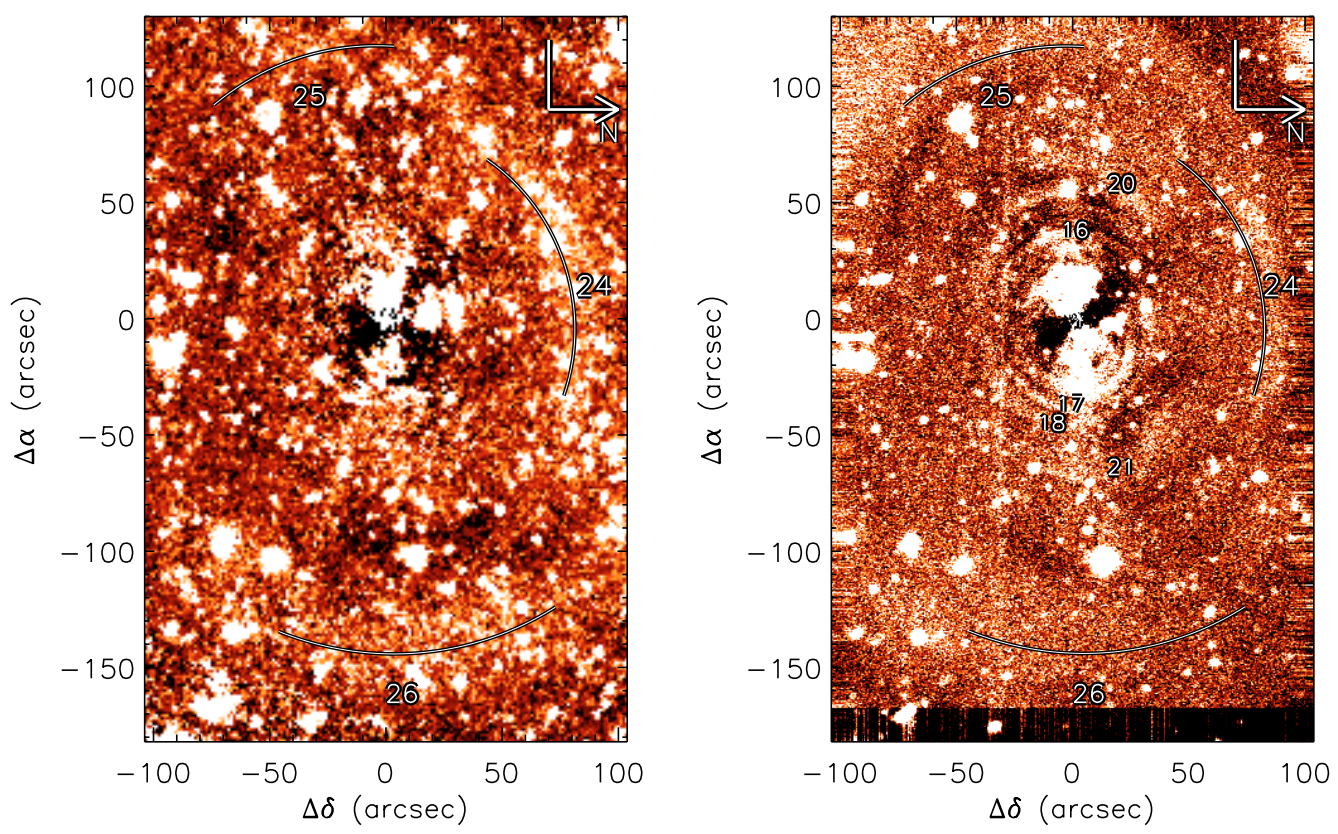

Fig. 4. Residual images of NGC 5982 at $3.6 \mu \mathrm{m}$ (left panel) and in the R-band (right). Some of the external shells are indicated by numbers following the notation of SCP07. The two newly discovered shells are 25 and 26 (see text).

Table 1. Surface photometry at $3.6 \mu \mathrm{m}$ and $4.5 \mu \mathrm{m}$.

\begin{tabular}{cccccl}
\hline \hline $\begin{array}{c}\lambda \\
\mu \mathrm{m}\end{array}$ & $\begin{array}{c}\mu_{\mathrm{e}} \\
\mathrm{mag} \mathrm{arcsec}\end{array}$ & $n$ & $\begin{array}{c}r_{\mathrm{e}} \\
\operatorname{arcsec}\end{array}$ & $N$ & $\begin{array}{l}\mathrm{rms} \\
\mathrm{mag} \mathrm{arcsec}^{-2}\end{array}$ \\
\hline 3.6 & $18.18 \pm 0.04$ & $5.8 \pm 0.2$ & $31.9 \pm 0.6$ & 79 & 0.040 \\
4.5 & $18.34 \pm 0.04$ & $5.7 \pm 0.2$ & $33.3 \pm 0.6$ & 79 & 0.042 \\
\hline
\end{tabular}

aperture corrections. The excess emission at $4.5 \mu \mathrm{m}$ is more elongated that that at 5.8 and $8 \mu \mathrm{m}$ (see Fig. 2), and represents $\sim 1 \%$ and $\sim 5 \%$ of the total emission at this wavelength when using the PAFW04 and RMC05 aperture corrections, respectively. The excess emission in the centre is significantly fainter, and is undetectable when using the RMC05 aperture corrections. We discuss in Sect. 5.1 the possible cause of the observed decrease in the excess emission towards smaller radii.

The [5.8]-[8.0] colour of NGC 5982 for the total emission is moderately red. Once the dominant stellar emission is subtracted, the [5.8]-[8.0] colour of the excess component is redder at smaller radii. If using the PAFW04 aperture corrections, the [5.8]-[8.0] colour of the excess emission approaches the values found in the star-forming galaxy M 81 (Willner et al. 2004) for $a \leq 20^{\prime \prime}$, and it reaches a value as high as $1.8 \pm 0.3 \mathrm{mag}$ at $a \sim$ $1.2^{\prime \prime}$. A value of $2.06 \mathrm{mag}$ is predicted by Li \& Draine (2001) for the Polycyclic Aromatic Hydrocarbon (PAH) emission, for a range of 5 orders of magnitude for UV radiation field. However, the [5.8]-[8.0] colour in the inner region is significantly less red when using the aperture corrections of RMC05 (see Fig. 3g). The [5.8]-[8.0] colour profile of NGC 4203 (PAFW04) is also sentitive to the aperture correction and shows a similar trend to NGC 5982 when using the same aperture correction.

\subsection{Colour gradients: underlying galaxy}

The mid-infrared colours provide information about the mean spectral type of the composite stellar population in the galaxy. The [3.6]-[4.5] colour (see Fig. 3e) is redder towards larger radii in NGC 5982. The colour change is comparable to a small change in the stellar population among $\mathrm{K}$ giant stars, typical for early-type systems. The [3.6]-[4.5] colour for the outer regions is similar to that of KOIII stars. Towards inner regions the [3.6]-[4.5] colour approaches values corresponding to K2-M0III stars.

The [3.6]-[8.0] colour is nearly constant in the inner bulge and similar to that found by PAFW04 in NGC 4203. However, NGC 5982 does not present the same strong gradient from $a \sim$ $60^{\prime \prime}$. The colour gradient from $a \sim 40^{\prime \prime}$ is very sensitive to the subtracted sky background at $8 \mu \mathrm{m}$.

\subsection{Warm and cold dust}

The dust carriers responsible for the emission at $24 \mu \mathrm{m}$ follow the same distribution as the stellar component emitting at $3.6 \mu \mathrm{m}$, which suggests that the dust is circumstellar. Emission at $24 \mu \mathrm{m}$ in the diffuse ISM of the Milky Way is due to very small grains (Léger \& Puget 1984; see also review of Draine 2003). At $18 \mu \mathrm{m}$ there is a broad silicate feature which is produced by Asymptotic Giant Branch (AGB) stars in ellipticals (Bregman et al. 2006). The emission images of NGC 5982 at 70 and $160 \mu \mathrm{m}$ reveal a different distribution for the corresponding carriers at these wavelengths from those responsible for the emission at shorter wavelengths. Figure 5 shows the $160 \mu \mathrm{m}$ emission profile along axis $\mathrm{PA}=121^{\circ}$ together with the $3.6 \mu \mathrm{m}$ emission profile smoothed to the same angular resolution.

The lack of correspondence between the 70 and $160 \mu \mathrm{m}$ images hinders the estimation of dust temperature. The bulk of the dust is traced by the $160 \mu \mathrm{m}$ emission. The carriers at this wavelength are likely to be large cold silicate grains which may have ice mantles (see, e.g., Draine 2003). The peak emission of warmer silicate grains will move towards shorter wavelengths. In the Milky Way emission at $70 \mu \mathrm{m}$ mostly traces very small grains (Draine 2003). If the dust emitting at $160 \mu \mathrm{m}$ has a temperature of $\sim 20 \mathrm{~K}$ and properties similar to those found in most elliptical galaxies from ISO data (Temi et al. 2004) we estimate a dust mass of a few times $10^{5} M_{\odot}$. This is in the mass range 


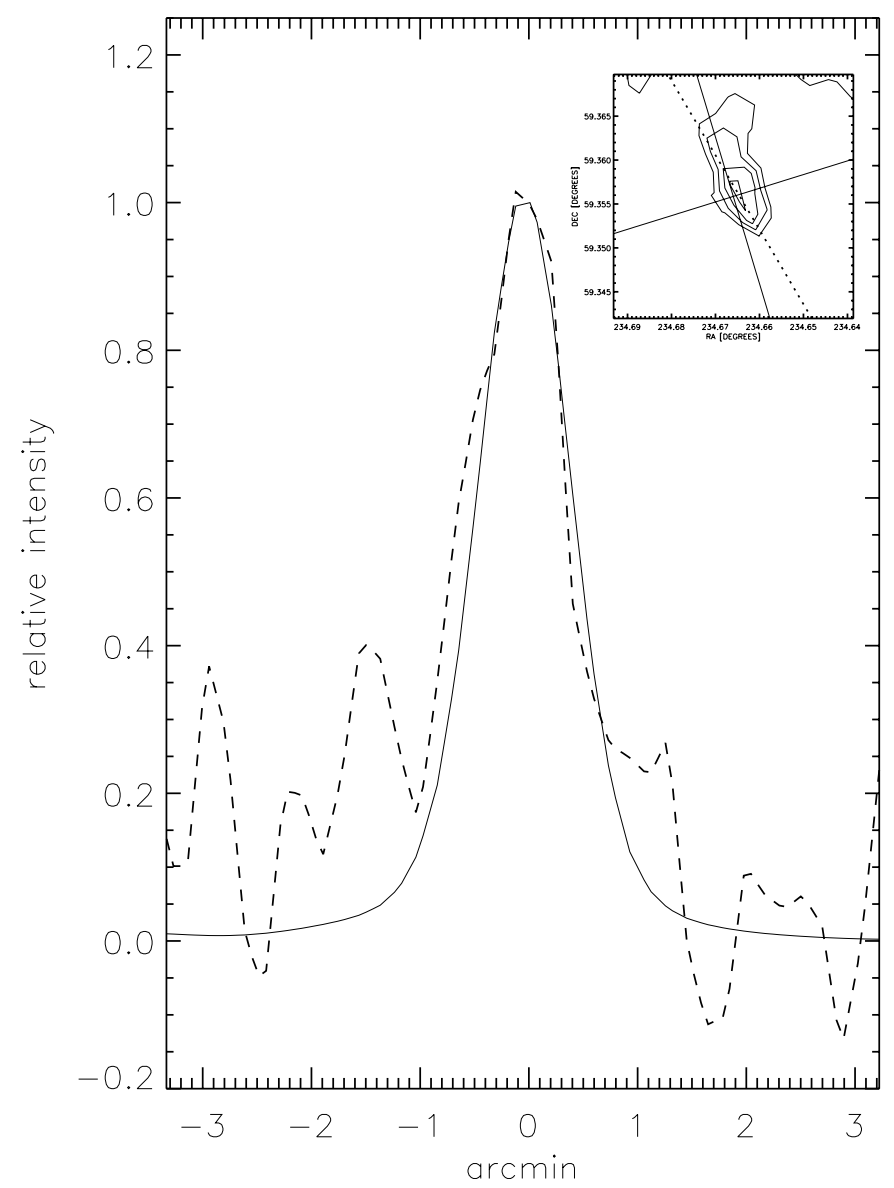

Fig. 5. Emission profile at $160 \mu \mathrm{m}$ (dashed line) and $3.6 \mu \mathrm{m}$ (continuous line) along an axis with $\mathrm{PA}=121^{\circ}$ passing through the $160 \mu \mathrm{m}$ emission peak. The $3.6 \mu \mathrm{m}$ emission profile has been smoothed to the angular resolution of the $160 \mu \mathrm{m}$ emission profile. At top-right is a map at $160 \mu \mathrm{m}$ with the minor and major axis plotted as continuous lines, and the $\mathrm{PA}=121^{\circ}$ axis as a dotted line.

derived from Spitzer data for a sample of ellipticals (Temi et al. 2007).

At $160 \mu \mathrm{m}$ we find a bright extended source with a peak emission of $4.8 \mathrm{MJy} \mathrm{sr}^{-1}$, spatially co-incident within the errors with 2MASX J15383273+5924022 from the 2MASS catalog of extended sources (Cutri \& Skrutskie 1998). This has counterparts at 3.6, 4,5, 5.8 and $24 \mu \mathrm{m}$ according to the images analyzed here. No redshift is available for this source, but it may well be in the background.

\subsection{Shells}

Shell structures are visible in the $3.6 \mu \mathrm{m}$ residual image (see Fig. 4, left). These correlate well with the shells found in SCP07, in particular in the ground-based $R$ band image shown in their Fig. 5 and our Fig. 4 (right). Below 40 arcsec radius, due to the lower angular resolution of the IRAC images, the shells are not as clear as in the HST/ACS $V$ - and $I$-band data.

In general, the shells are much fainter in the $4.5 \mu \mathrm{m}$ residual image than in the $3.6 \mu \mathrm{m}$ residual image. We can not distinguish any shells at $5.8 \mu \mathrm{m}$. To the best of our knowledge this is the first time that shells are observed in the mid-infrared.

We have discovered two probable outer shells from the midinfrared emission, labelled 25 and 26 (see Fig. 4 left) which are not visible in the HST/ACS images (shell 26 is mostly outside the field of view), although they do appear to be visible in the ground-based $R$-band image (see Fig. 4 right). These shells are circular arcs, located at distances of about 126" (shell 25 ) and $150^{\prime \prime}$ (shell 26) on opposite sides along the major axis. Shell 24, the outermost shell detected in the HST/ACS images, is clearer in both the 3.6 image and the $R$-band image. This is the only shell to lie on the minor axis of this galaxy.

Using the methodology outlined in SCP07, we determine the luminosity profile of shell 24 by performing surface photometry in circular and elliptical wedges, of a constant opening angle chosen to fully include the azimuthal extent of the shell, centred on the photometric centre of the galaxy, and extending to beyond the shell radius. Figure 6 (left) shows the resulting surface brightness profiles at 3.6 and $4.5 \mu \mathrm{m}$ of shell 24 using circular wedges. Figure 6 (right) shows the residuals (i.e., difference between the observed surface brightness and the galaxy model extracted within wedges) versus radius (i.e., distance from the photometric centre) at 3.6 and $4.5 \mu \mathrm{m}$ as well as in the $R$-band for shell 24 . The shell profiles are very similar at these wavelengths and are in good agreement with those derived from HST/ACS $V$ - and $I$-band data.

An analysis of the shell profiles to learn about their 3D density distribution is carried out using the methodology described in SCP07. In the first model, it is assumed that the shell has an intrinsic $\left(r_{0}-r\right)^{-0.5}$ density distribution, where $r_{0}$ is the radius of maximum stellar density. This model is predicted by theory to describe the inner parts of phase wrapped shells (Dupraz \& Combes 1986; Prieur 1988). In the second model, it is assumed that shells have an intrinsic Gaussian shape with thickness $r_{\mathrm{g}}$. Although this model lacks a physical basis, it has been used to describe spatially wrapped shells (Turnbull \& Collet 1999). Figure 7 shows the model fits for the $V$-band and at $3.6 \mu \mathrm{m}$. The Gaussian model is a better fit to the observations at all the wavelengths. The first model suffers from "noise" and a sudden drop in flux at $r_{0}$ due to its discontinuous nature, and to the noise in the data (see SCP07).

The mean colours of shell 24 are obtained. The [3.6]-[4.5] colour of shell 24 is determined using both circular and elliptical wedges. In each case we find a consistent mean colour difference between the shell and the underlying galaxy of $0.05 \pm 0.01 \mathrm{mag}$, in the sense that the shell is bluer than the underlying galaxy. The mean colour of the underlying galaxy is [3.6]-[4.5] = $-0.08 \mathrm{mag}$ at the shell position. The $V-I$ colour of this shell and the underlying galaxy at this position are also determined. We find that that $V-I$ is $1.16 \pm 0.10 \mathrm{mag}$ and $\sim 1.25 \mathrm{mag}$ for the shell and the underlying galaxy respectively, indicating that the shell is also bluer than the galaxy at optical wavelengths.

\section{Discussion}

\subsection{Stellar populations: underlying galaxy}

The stellar population in NGC 5982 is dominated by old stars. The galaxy is classified as type YP, i.e., containing a young stellar population mixed with the old one, by Michard \& Prugniel (2004). Mc Dermid et al. (2006) found that the stellar population in the inner $2 \mathrm{kpc}$, corresponding to the size of the KDC, has a luminosity weighted mean age of $\sim 8$ Gyr. Mc Dermid et al. also claim that the KDC is unlikely to be the result of a recent merger, although formation from a recent "dry" merger is not ruled out. Sikkema et al. (2006) observed that the globular cluster populations in NGC 5982 are consistent with an old (>5 Gyr) and evolved system. We find that the surface brightness profile at $3.6 \mu \mathrm{m}$ resembles that corresponding to the HST/ACS 

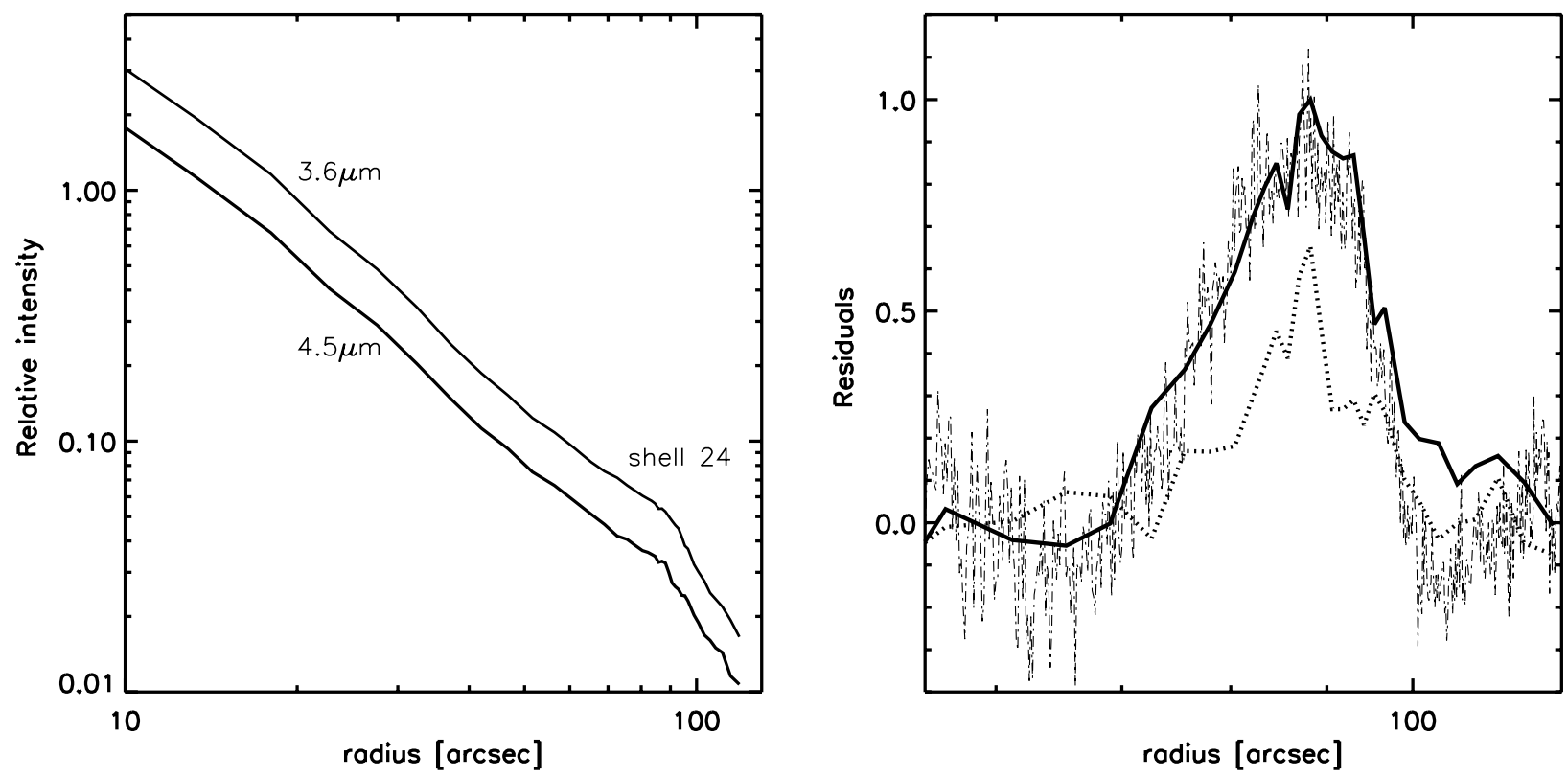

Fig. 6. Left: mean surface luminosity in circular wedges with an opening angle of \pm 20 deg to sample the shell 24 at $3.6 \mu \mathrm{m}$ and $4.5 \mu \mathrm{m}$. Right: residuals (i.e., observed emission minus galaxy model in circular wedges) at $3.6 \mu \mathrm{m}$ (continuous line) and $4.5 \mu \mathrm{m}$ (dashed line) and in the $R$-band (dash-dotted line) versus radius. Residuals are relative: those in the $R$-band and at $3.6 \mu \mathrm{m}$ have been re-scaled for a proper comparison. The scaling factor applied to the residuals at 3.6 and $4.5 \mu \mathrm{m}$ is the same.
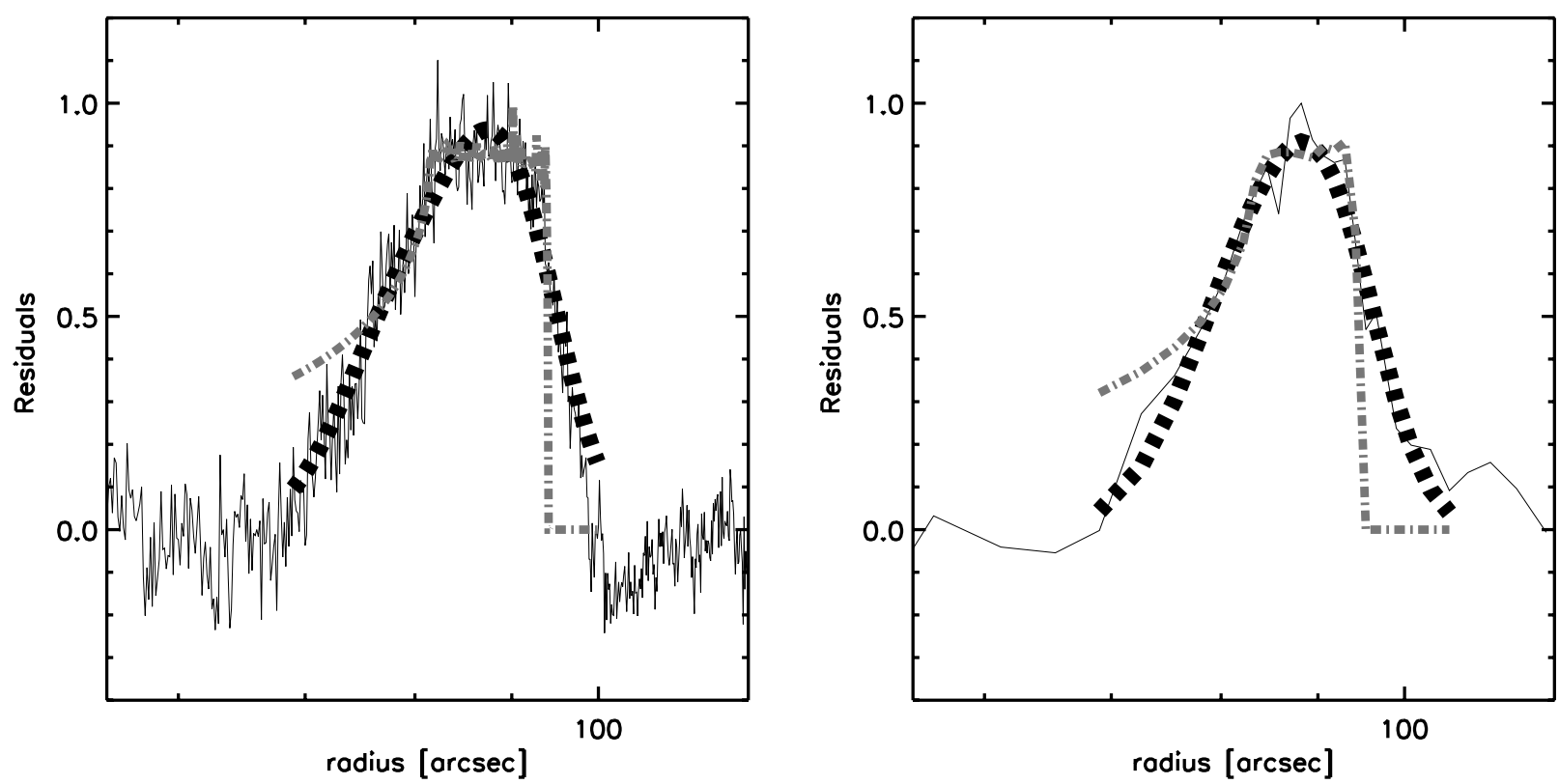

Fig. 7. Shell profiles in the $V$-band (left) and at $3.6 \mu \mathrm{m}$ (right) with the Gaussian distribution fit (thick dashed line) and symmetrical density model (dot-dashed line).

I-band image. This indicates that the emission from old stellar photospheres dominates the bulk emission at these wavelengths. This further supports our assumption that the $3.6 \mu \mathrm{m}$ emission is entirely photospheric and unaffected by dust emission, and therefore it can be used as a stellar template to determine any excess emission at 4.5, 5.8 and $8.0 \mu \mathrm{m}$ (see Sect. 3.2). We find that the emission at $4.5 \mu \mathrm{m}$ is mostly photospheric, with a faint excess emission. The [3.6]-[4.5] colour is bluer towards the inner regions, with a profile that matches the colours of late $\mathrm{K}$ or $\mathrm{M}$ giants (see Fig. 3e). Inner regions could have a major contribution from low-mass AGB stars with lower mass-loss rates, which would explain their low excess to total emission ratios (see Sect. 4.1). Colour [3.6]-[8.0] is too red to be explained by any stellar population. This may be a signature of the extended excess emission detected in NGC 5982. A similar value for [3.6]-[8.0] was found in NGC 4203 by PAFW04 who interpreted it as due to warm dust emission at $8.0 \mu \mathrm{m}$.

\subsection{Dust populations}

Systematic uncertainties in the aperture corrections, especially at $5.8 \mu \mathrm{m}$, hinder the analysis and interpretation of the excess emission colours (see Sects. 3.2 and 4.1). The excess emission 
at $8 \mu \mathrm{m}$ obtained using the two aperture corrections is very similar. However, the ratio of excess to total emission at $5.8 \mu \mathrm{m}$ is considerably higher when using the aperture corrections of $\mathrm{RMC05}$ and it is more difficult to explain its origin since the emission below $8 \mu \mathrm{m}$ is expected to be dominated by stellar photospheres (Bressan et al. 2006). The mid-infrared SEDs of passive early-type galaxies contain emission from mass-losing oxygen-rich AGB stars, with a silicate emission feature at $10 \mu \mathrm{m}$ (Bressan et al. 1998, 2006; Bregman et al. 2006). Some excess emission at 5.8 and $8 \mu \mathrm{m}$ may originate in the moderately thick circumstellar envelopes of the AGB stars, where silicate dust grains are formed and heated to $\sim 300 \mathrm{~K}$ (Bregman et al. 2006). The IRAC filterband at $8 \mu \mathrm{m}$ is sensitive to the $9-12 \mu \mathrm{m}$ emission from silicates. At shorter wavelengths the continuum is produced near the stellar photosphere (Bregman et al. 2006). The lower excess emission observed in the centre would be due to a lower mass-loss rate from AGBs (see previous section).

Excess emission at 5.8 and $8 \mu \mathrm{m}$ may be due to PAH emission. There is a series of emission bands, emitting at 3.3, 6.2, 7.7, 8.6, 11.3, 12.7 and $16.4 \mu \mathrm{m}$, observed in interacting early type galaxies (NGC 4435: Panuzzo et al. 2006), starbursts and AGNs (M87: Bressan et al. 2006). Bands at 6.2, $7.7 \mu \mathrm{m}, 8.2$ and $8.6 \mu \mathrm{m}$ lie within the IRAC filterbands at 5.8 and $8 \mu \mathrm{m}$. The central [5.8]-[8.0] colour in NGC 5982 obtained using the aperture corrections of PAFW04 resembles that from PAH emission. This is not confirmed, however, when using the RMC05 aperture corrections.

The excess emission could be due to very warm dust heated by a central AGN. It is not clear, however, whether there is a central LINER in NGC 5982 (Ho et al. 1997). A low level AGN may influence its surroundings. UV radiation from an active nucleus could be hard enough to destroy PAHs and even very small grains. Only big grains would survive. The dust destruction by the AGN may be restricted, however, to a few hundred parsecs, i.e., a region well within the IRAC PSF. Sputtering by hot gas can affect regions much farther out in the galaxy. On the other hand, a depletion of small particles could be also produced by dust coagulation, with the smaller particles being adsorbed onto the surface of big grains, as is observed in dense regions of the Milky Way (see, e.g., Stepnik et al. 2003; del Burgo et al. 2003, and references therein).

The lack of definitive observations to determine the level of AGN activity makes our discussion more difficult. It is plausible that AGB mass-loss could largely be responsible from the extended excess emission in NGC 5982, as is observed in many other early-type galaxies (Bressan et al. 2006). This is also in agreement with 6.7 and $15 \mu \mathrm{m}$ extended emission observations in early-type galaxies (Xilouris et al. 2004).

We find similar surface brightness distributions at $3.6 \mu \mathrm{m}$ and $24 \mu \mathrm{m}$. This suggests that the $24 \mu \mathrm{m}$ emission is mostly due to circumstellar dust associated with the stellar component traced at $3.6 \mu \mathrm{m}$. In the diffuse ISM of our Galaxy emission at $24 \mu \mathrm{m}$ is interpreted as due to very small (a few nanometer in size) grains that are transiently heated by the stellar radiation field and have a peak emission at $\sim 60 \mu \mathrm{m}$. Such very small grains are likely to be the dust population traced by the $24 \mu \mathrm{m}$ emission in NGC 5982.

Big silicate grains can be traced by the Spitzer/MIPS filterbands at 70 and $160 \mu \mathrm{m}$. Temi et al. (2004) used a two dust temperature model to fit the far-infrared emission in a rather inhomogeneous ISO sample of 53 early-type galaxies. They found temperatures of around $40 \mathrm{~K}$ and $20 \mathrm{~K}$, the latter being the dominant component by mass. Generally, big grains would present a continuous temperature range. In dense/cold regions of the
Milky Way, the dust absorption/coagulation of small grains onto the surfaces of big grains with ice mantles is favoured. This results in grains with enhanced far-infrared emissivities with respect to the grains in the diffuse interstellar medium (Stepnik et al. 2003; del Burgo et al. 2003). The different surface brightness distributions observed in NGC 5982 at 70 and $160 \mu \mathrm{m}$ may be partly due to changes in the abundances and properties (temperature, emissivity) of dust. This far-infrared emitting dust, consisting of grains of various size and temperature, appears to be distributed in a disk inclined to the principal axes. If the average emissivity of the cold big grains were enhanced, as has been proposed to explain the SEDs in spirals (Dasyra et al. 2005), less dust would be necessary to account for the far-infrared emission.

The surface brightness distributions at 70 and $160 \mu \mathrm{m}$ suggest a dust source additional to mass-loss from the underlying stellar population. In Sect. 5.4 we discuss the origin of this dust.

\subsection{Origin of shells}

Shells in NGC 5982 are expected to be the result of phase wrapping. The well defined type I shells in NGC 5982 are thought to be created by a minor merger with a small, non-rotating galaxy.

The large number of shells found in NGC 5982 indicates an old age. We have revised the dynamical age of the shells in this galaxy found by SCP07 taken into account our discovery of the outermost shells. The dynamical age, estimated from Eq. (88) of Nulsen (1989), using the outer shell radii found in this paper, and assuming a mass for NGC 5982 of $4.57 \times 10^{11} M_{\odot}$ from the fundamental plane method (Eq. (7) of van Dokkum \& Stanford $2003)$ is $1.1 \times 10^{9}$ years.

We analyze the colours [3.6]-[4.5] and $V-I$ in the shell 24, the only one found on the photometric minor axis. Both colours of the shell are bluer with respect to the underlying galaxy. Blue shells may indicate the presence of a younger stellar population, not necessarily confined to the shells themselves. It may also be related to the presence of a significant metal-poor component within the shell. This hypothesis is supported by the positive correlation between metallicity and galaxy mass (Sandage \& Visvanathan 1978) and the expected much smaller mass of the galaxy intruder with respect to the main galaxy. Finally, it could be that the shells contain less dust per unit stellar mass than the underlying galaxy or that the dust in the shells has different properties. It is suggestive that the [3.6]-[4.5] in the shell 24 resembles that in the central region of NGC 5982 where a faint excess emission is observed. Blue $V-I$ colours have been found in other shell ellipticals, for example, in the South side of NGC 1344, which may be evidence for a relatively recent merger event (SCP07).

Stickel et al. (2004) claimed to find a large amount $(7 \times$ $\left.10^{4} M_{\odot}\right)$ of cold $(T \approx 15 \mathrm{~K})$ dust associated with atomic and molecular gas in the northern shell of NGC 5128. We observe only some extended and extremely faint emission at $160 \mu \mathrm{m}$ (surface brightness of $\approx 0.5 \mathrm{MJy} \mathrm{sr}^{-1}$ above the sky background) at the position of shell 24 . We do not find any excess emission at $4.5,5.8$ or $8.0 \mu \mathrm{m}$ associated with this shell. We do find, however, a counterpart in the mid-infrared for the HI gas cloud found by Morganti et al. (2006), which is close to the shell 13 found by SCP07 (see Sect. 5.5).

\subsection{Origin of cold dust}

The far-infrared emission in ellipticals is not entirely explained by steady state models of dust production by mass loss from the 
old population of red giant stars and dust destruction by grain sputtering in the hot interstellar gas (Temi et al. 2007). The excess of cold dust could have an external origin, from a recent merger with another galaxy. However, Temi et al. (2007) argue that the most plausible origin of the far IR emission is an internal mechanism, whereby cold dust originating from mass-loss from stars in the core is accumulated, and then buoyantly transported to distances of a few kpc by intermittent energy outbursts from AGNs.

More precise spectroscopic observations are required to verify or exclude the existence of a low-level AGN in the nucleus of NGC 5982, and to quantify the impact on the origin of the excess emission.

NGC 5982 contains warm gas (traced by $\mathrm{H} \beta$, [OIII] $\lambda 5007$ ) in the central $4 \mathrm{kpc}$ that lies in a rapidly rotating central component and in a filament extending from the core to the Southern side (Sarzi et al. 2006). The ionized gas in the centre rotates in the same sense as the stars and there is a velocity gradient along the filament, with positive velocities in the Southern edge and negative velocities in the innermost part. Temi et al. (2007) found that optical emission line fluxes from warm gas correlate weakly with $L_{70}$ and $L_{160}$ in ellipticals, suggesting that the dust may be responsible for cooling the warm gas. They also suggest that the warm gas has an internal origin, being expelled by orbiting red giant stars. Both results are, however, not definitive due to the poor quality of the dataset. In NGC 5982 we find that the spatial distributions of the warm gas and the dust are different. There is no dust emission along the filament of warm gas. In the central $2.5 \mathrm{kpc}$ region, the warm gas lies on some faint $70 \mu \mathrm{m}$ emission, and it encloses the irregularly distributed patches traced by optical absorption (SCP07). There is also a small patch of warm gas which lies on the southern peak of the $70 \mu \mathrm{m}$ emission. Dusty warm gas forms possibly optically thick central disks in ellipticals (Lauer et al. 2005; Sarzi et al. 2004). The observations of SCP07 in a sample of shell ellipticals indicate that the dust seems to be out of dynamical equilibrium, forming irregular patches and lanes. SCP07 conclude that shell ellipticals have a higher dust prevalence than normal early type galaxies and that external influences are responsible for the ubiquitous presence of dust.

No HI gas is found in the central region of NGC 5982. In the next section we describe in more detail the HI cloud found by (Morganti et al. 2006).

In the internal dust origin scenario of Temi et al. (2007), cooling to below $10^{4} \mathrm{~K}$ and consequent molecular formation are expected. Conversely, Combes et al. (2006) suggest that the $\mathrm{CO}$ emission found in some ellipticals has been accreted in many of them, and that it is the material fueling star formation. No CO is detected in the central region of NGC 5982, with beam resolutions of $F W H M \sim 23$ and $12 \operatorname{arcsec}$ (Combes et al. 2006). We note, however, that the upper limit for the $\mathrm{H}_{2}$ gas of $3.7 \times 10^{7} M_{\odot}$ (Combes et al. 2006) is approximately two orders of magnitude above our estimated cold dust mass. On the other hand, the $\mathrm{CO} / \mathrm{H}_{2}$ ratio is very uncertain and the $\mathrm{H}_{2}$ content may be underestimated by enhanced photodissociation of the $\mathrm{CO}$ molecule.

The central cold dust emission traced at 70 and $160 \mu \mathrm{m}$ represents the bulk of dust in NGC 5982. If the dust seen in Figs. $1 \mathrm{~g}$ and $\mathrm{h}$ forms a disk inclined to the principal axes of NGC 5982 then it would be expected to precess to the major axis on a timescale of between 0.5 and 2 times the precession timescale (Steiman-Cameron \& Durisen 1990). The simulations of Varnas (1990) suggest that this will happen in about $0.5-1.0 \times 10^{9}$ years. If the dynamical age (see Sect. 5.3) is greater than the precession time then we would not expect to see the dust morphology found, however both of these estimated ages have uncertainties of at least a factor two, and we cannot conclude that they are inconsistent. The dust survival time (i.e., sputtering lifetime) should be also consistent. It is expected to be low in the centres of early type galaxies $\left(\sim 10^{8} \mathrm{Gyr}\right)$ although the self-shielding in dust clouds may increase the estimates considerably (SCP07). If the sputtering lifetime is much shorter than the merger age and the dust has a external origin, then it was accreted in the late stages of the merger or in a more recent merger.

\subsection{HI gas cloud}

There is a small ( $\approx 4 \mathrm{kpc}$ in size) HI cloud located $\sim 6 \mathrm{kpc}$ to the ENE of the optical photometric centre of the galaxy, with a mass of $3.4 \times 10^{7} M_{\odot}$ and at a velocity of $2830 \mathrm{~km} \mathrm{~s}^{-1}$, only $\sim 200 \mathrm{~km} \mathrm{~s}^{-1}$ lower than the systemic velocity (Morganti et al. 2006). The HI cloud is close to the shell 13 found in SCP07, located 27.3" (i.e., $5.7 \mathrm{kpc}$ ) E. We find emission at 3.6, 4.5, 5.8, 24 and $70 \mu \mathrm{m}$ which is very close to the centre of the HI beam (see Fig. 8, top and bottom). There is no ionized gas detected on the HI cloud although the ionized gas velocities are consistent with the HI velocity (Morganti et al. 2006).

If the HI gas and cold dust observed in NGC 5982 have the same origin, their very different spatial distributions observed today suggest a severe disruption of these components. We note that the dust mass estimated from the HI gas cloud is, dependent upon the assumed gas-to-dust ratio, of a few times $10^{5} M_{\odot}$, a value of the same order as our estimated dust mass (see Sect. 4.3). A search for $\mathrm{CO}$ emission at the position of the HI cloud would be valuable.

The very different distributions of the ISM components, the presence of a KDC (Wagner et al. 1988) and shells, and the proximity of other galaxies around NGC 5982 (García 1993) suggest a merger scenario to explain the shell formation and the origin of dust in this galaxy.

\section{Conclusions}

We have performed a surface photometry study of the underlying galaxy and shells in NGC 5982 from Spitzer IRAC and MIPS filterband data. Our conclusions are the following:

- Shells are clearly detected from their stellar emission at 3.6 and $4.5 \mu \mathrm{m}$. To the best of our knowledge this is the first time shells are observed in the mid-infrared. In addition to the optical shells noted by SCP07, we have discovered two most external shells at larger radius. Most shells are regularly distributed on both sides along the photometric major axis. Only shell 24 is in the perpendicular direction. We find this shell to be bluer in [3.6]-[4.5] and $V-I$ colours than the underlying galaxy. The shell profiles in the optical, near-infrared and mid-infrared are alike and well fitted with a Gaussian shape model. The newly discovered shells cause a revision of the estimate of the dynamical age to $\sim 1.1 \times 10^{9}$ years.

- Following subtraction of the stellar contribution, excess emission is observed at 4.5, 5.8 and $8.0 \mu \mathrm{m}$. The $4.5 \mu \mathrm{m}$ emission excess is very faint and concentrated in a disk-like structure extending from the centre of the galaxy. The emission excess at 5.8 and $8.0 \mu \mathrm{m}$ is extended and possibly traces dust originating from stellar mass loss.

- The distributions of surface brightness at $24 \mu \mathrm{m}$ and $3.6 \mu \mathrm{m}$ are very similar. This shows that the dust emitting at $24 \mu \mathrm{m}$ has a circumstellar origin. 

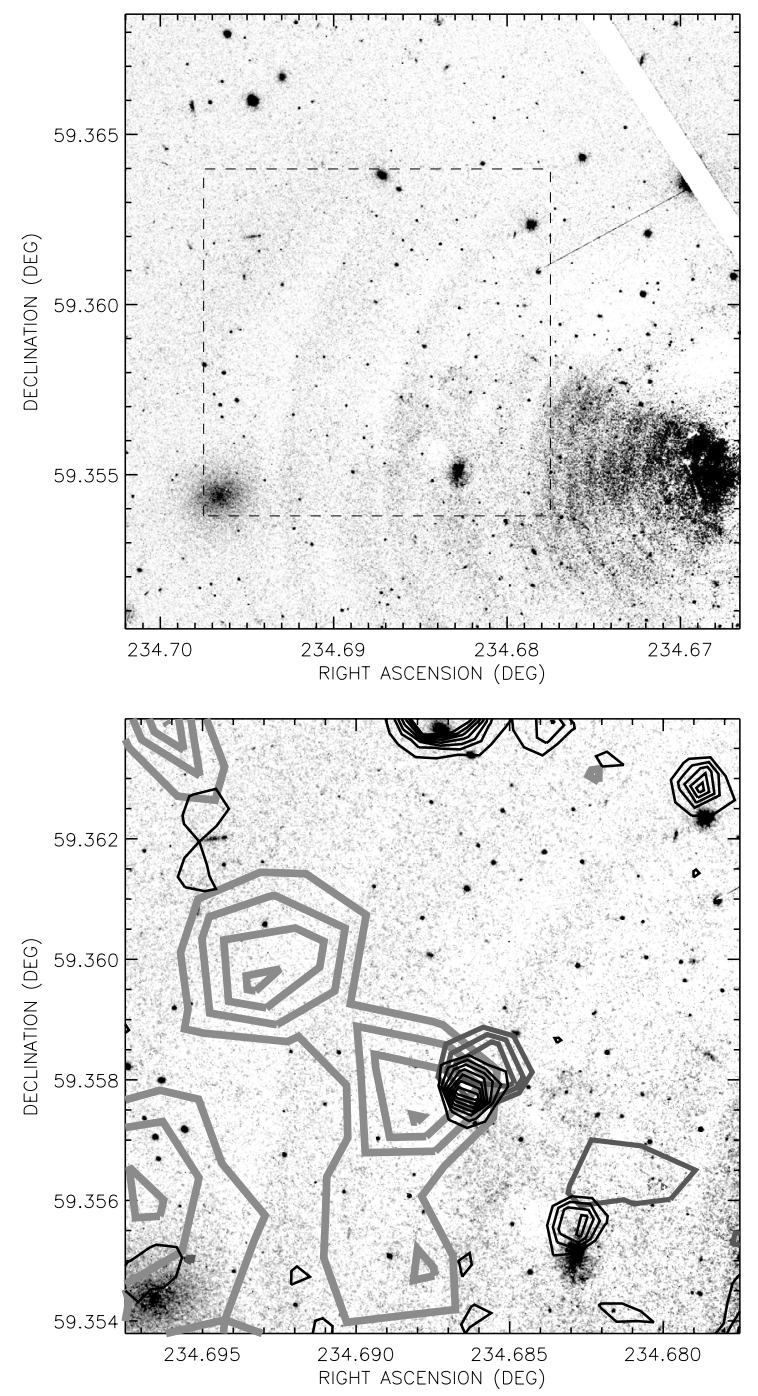

Fig. 8. HST/ACS $V$-band image (top). The centre of the image corresponds to the position of the HI gas cloud detected with a beam of $36^{\prime \prime} \times 36^{\prime \prime}$ (Morganti et al. 2006). The region inside the square in dashedline is zoomed (bottom) with 3.6, 24 and $70 \mu \mathrm{m}$ emission contours (thin, thick and double-thick lines, respectively).

- Longer wavelength emission due to larger and colder dust grains is more confined to the inner regions of the galaxy. Warm dust traced by the $70 \mu \mathrm{m}$ image has a double peak a few Kiloparsec to the Northern side of the galaxy, on the opposite side to the [OIII] $\lambda 5007$ peak detected by Sarzi et al. (2006). Cold dust traced by the $160 \mu$ m emission appears to form a few (7-8) kpc radius disk, and represents the bulk of the dust mass. It has a mass of a few $10^{5} M_{\odot}$, approximately three orders of magnitude higher than that deduced from optical obscuration alone, but consistent with what might be associated with a small HI cloud on the Eastern side detected by Morganti et al. (2006).

- The very different distributions of the HI gas, ionized gas, optical and cold dust and the presence of shells and a KDC in NGC 5982 support a merger scenario in this galaxy.

Acknowledgements. We acknowledge the anonymous referee for his/her useful comments. This work is based on observations made with the Spitzer Space Telescope, which is operated by the Jet Propulsion Laboratory, California
Institute of Technology, under a contract with NASA. Also it is based on observations made with the NASA/ESA Hubble Space Telescope, obtained at the Space Telescope Institute, which is operated by the Association of Universities for research in Astronomy, Inc., under NASA contract NAS 5-26555. These observations are associated with program GO9399.

\section{References}

Bregman, J. N., Temi, P., \& Bregman, J. D. 2006, ApJ, 647, 265 Bressan, A., Granato, G. L., \& Silva, L. 1998, A\&A, 332, 135

Bressan, A., Panuzzo, P., Buson, L., et al. 2006, ApJ, 639, 55

Caon, N., Capaccioli, M., \& D’Onofrio, M. 1993, MNRAS, 265, 1013

Carter, D. 1978, MNRAS, 182, 797

Combes, F., Young, L., \& Bureau, M. 2007, MNRAS, 377, 1795

Cutri, R. M., \& Skrutskie, M. F. 1998, BAAS, 30, 1374

Dasyra, K. M., Xilouris, E. M., Misiriotis, A., \& Kylafis, N. D. 2005, A\&A, 437, 447

del Burgo, C., Laureijs, R., Ábrahám, P., \& Kiss, Cs. 2003, MNRAS, 346, 403

Dupraz, C., \& Combes, F. 1986, A\&A, 166, 53

Draine, B. T. 2003, ARA\&A, 41, 241

Emsellem, E., Cappellari, M., Peletier, R. F., et al. 2004,

Fazio, G. G., Hora, J. L., Allen, L. E., et al. 2004, ApJS, 154, 10

Ferrari, F., Pastoriza, M. G., Macchetto, F. D., et al. 2002, A\&A, 389, 355

Frogel, J. A., Persson, S. E., Matthews, K., \& Aaronson, M. 1978, ApJ, 220, 75

García, A. M. 1993, A\&AS, 100, 47

Hernquist, L., \& Quinn, P. J. 1988, ApJ, 312, 17

Ho, L., Filippenko, A. V., \& Sargent, L. W. 1997, ApJS, 112, 315

Jørgensen, I., Franx, M., \& Kjaergaard, P. 1992, A\&AS, 95, 489

Kaneda, H., Onaka, T., \& Sakon, I. 2005, ApJ, 632, 83

Knapp, G. R., Guhathakurta, P., Kim, D.-W., \& Jura, M. A. 1989, ApJS, 70, 329

Lauer, T. R., Faber, S. M., Gebhardt, K., et al. 2005, AJ, 129, 2138

Léger, A., \& Puget, J.-L. 1984, A\&A, 137, L5

Li, A., \& Draine, B. T. 2001, ApJ, 554, 778

Malin, D. F., \& Carter, D. 1980, Nature, 285, 643

Malin, D. F., \& Carter, D. 1983, ApJ, 274, 534

Matthews, W. G., \& Brighenti, F. 2003, ApJ, 599, 992

McDermid, R. M., Bacon, R., Kuntschner, H., et al. 2006, NewAR, 49, 521

Michard, R., \& Prugniel, P. 2004, A\&A, 423, 833

Morganti, R., de Zeeuw, P. T., Oosterloo, T. A., et al. 2006, MNRAS, 371, 157

Nulsen, P. E. J. 1989, ApJ, 346, 690

Pahre, M. A., Ashby, M. L. N, Fazio, G. G., \& Willner, S. P. 2004, ApJS, 154, 229 (PAFW04)

Panuzzo, P., Vega, O., Bressan, A., et al. 2007, ApJ, 656, 206

Pavlovsky, C., et al. 2004, ACS Data Handbook, Version 3.0 (Baltimore: STScI) Prieur, J.-L. 1988, Ph.D. Thesis, Toulouse: Universite Sabatier, Observatoire Midi-Pyrénées

Quinn, P. J. 1984, ApJ, 279, 596

Reach, W., Megeath, S. T., Cohen, M., et al. 2005, PASP, 117, 978 (RMC05)

Rieke, G. H., Young, E. T., Engelbracht, C. W., et al. 2004, ApJS, 154, 25

Sandage, A., \& Visvanathan, N. 1978, ApJ, 223, 707

Sarzi, M., Falcón-Barroso, J., Davies, R. L., et al. 2006, MNRAS, 366, 1151

Sersic, J.-L. 1968, Atlas de Galaxias Australes (Cordoba: Observatorio Astronomico)

Sikkema, G., Peletier, R., Carter, D., Valentijn, E. A., \& Balcells, M. 2006, A\&A, 458,53

Sikkema, G., Carter, D., Peletier, R., et al. 2007, A\&A, 467, 1011, SCP07

Smith, R. J., Lucey, J. R., Hudson, M. J., Schlegel, D. J., \& Davies, R. L. 2000, MNRAS, 313, 469

Steiman-Cameron, T. Y., \& Durisen, R. H. 1990, ApJ, 357, 62

Stepnik, B., Abergel, A., Bernard, J.-P., et al. 2003, A\&A, 398, 551

Stickel, M., van der Hulst, J. M., van Gorkom, J. H., Schiminovich, D., \& Carilli, C. L. 2004, A\&A, 415, 95

Temi, P., Brighenti, F., Mathews, W. G., \& Bregman, J. D. 2004, ApJS, 151, 237

Temi, P., Brighenti, F., \& Mathews, W. G. 2007, ApJ, 660, 1215

Turnbull, A. J., \& Collett, J. L. 1999, ASPC, 182, 485

van Dokkum, P. G., \& Franx, M. 1995, AJ, 110, 2027

van Dokkum, P. G., \& Stanford, S. A. 2003, ApJ, 585, 78

Varnas, S. R. 1990, MNRAS, 247, 674

Wagner, S. J., Bender, R., \& Möllenhoff, C. 1988, A\&A Lett., 195, 5

Werner, M. W., Roelling, T. L., Low, F. J., et al. 2004, ApJS, 154, 1

Willner, S. P., Ashby, M. L. N., Barmby, P., et al. 2004, ApJS, 154, 222

Xilouris, E. M., Madden, S. C., Galliano, F., Vigroux, L., \& Sauvage, M. 2004, A\&A, 416, 41 